\title{
Air Pollution due to Desertification and Diseases Caused by It
}

\author{
Nooshin Shakerian ${ }^{1}$, Hasan khosravi ${ }^{1}$, Behzad Shakerian \\ ${ }^{1}$ Tehran University \\ Iran \\ n.shakerian@ut.ac.ir
}

\begin{abstract}
Pollution is one of the most dangerous threats to humans and even animals. Desertification and climate change are the most important environmental challenges in arid regions, which affects the health of people all over the world such as air pollution. Air pollution has become a global problem today, which threatens the health of humans around the world. Air pollution although is harmful for all people of all ages but a wide range of people, including children, the elderly, pregnant women and patients are more vulnerable than others. Most of the pollution caused by air pollution is related to the respiratory system and lungs, the immune system, the heart, and the vision system. In the study of air pollutants and dust particles, dust and greasers are significant parts. In this research, we tried to explain about these materials a little and how they are produced, their mode of action, the diseases cause by them and the ways to prevent these harmful pollutants. Also we studied the climatic parameters (precipitation, temperature, evapotranspiration and wind) to determine dry month of area and the effect of it in Khuzestan province (IRAN) that is affected by dust since 2003. Based on the results of the most important climatic factors that influence desertification and air pollution of the Khuzestan the month of April to mid-October, the region was dry, so that, June, July and August seems to be its greatest extent. The prevalence of diseases caused by dust and air pollution is the most in these months. This study was prepared with the help of a group of Iranian medical professors in a descriptive method.
\end{abstract}

Keywords: Air pollution, Particulate matter, Disease, Wind erosion, Dust.

\section{Introduction}

In addition to the cars in the streets and the activities of industrial factories and their pollutants, the desert phenomenon and desertification process in many parts of the world, especially in the arid and semi-arid regions of the world, have attracted the attention of many experts and researchers in the pollution sector.

Reducing of biological and ecological potential of lands due to inappropriate climatic conditions, rainfall shortages or destructive effects of mankind and its degradation and manipulation in nature leads to the process of desertification and erosion. Desertification and erosion, in addition to their effects on reducing the soil and land fertility, cause very dangerous environmental pollution, such as air pollution, which includes dust, dust and droplet contamination, which greatly affects the health of humans, animals and other organisms. This destructive phenomenon during short or long contact with people causes a wide range of cardiovascular, respiratory, allergic diseases, cancers and many unknown diseases. Dusts are caused by wind erosion. Global warming, low rainfall, high evapotranspiration, relatively high wind speeds, are effective in increasing of this trend. Activities such as over-exploitation of groundwater, grazing of the livestock in the slopes, unprocessed harvesting of mines and etc. cannot be ignored in these areas.

\section{Types of Dust and Its Effects on Human Health}

Dust is either a mass fraction of solid particles of fine soil or sometimes smoke that diffuses in the atmosphere which limits the horizontal visibility of about 1 to 2 kilometres. There are various studies conducted around the world on suspended particles in the air, in particular the effects of dust and fogs on human health. These researches show that these particles are divided into several groups according to their size [10]:

1. Particles with a diameter of less than $0.01 \mu \mathrm{m}$ (UFP)

2. particles with a diameter of less than $2.5 \mu \mathrm{m}$

3. particles with a diameter of 2.5 to 10 micrometres

For finding the effect of these particles to human health, particles with a diameter of 2.5 to 10 micrometres have the highest levels of pathogenicity. Particles with smaller size remain suspended in the respiratory air and can enter and leave 
the lungs without getting tucked in the respiratory system. Particles larger than this range usually precipitate in the air purifying system of the upper respiratory tract (nose) and cannot enter the other breathing organs and lungs [11].

The concentration of suspended particles ranges from 2.5 to 10 micrometres Measure based on micrograms per cubic meter and UFP levels based on their number per cubic meter [10].

The contaminating particles may cause inflammatory reactions of the lungs or other chemical properties and react with the normal discharge of the body or tissues of the respiratory tract. These particles may also carry other biological particles that are harmful to health, such as Fungal spores or pollen, or the activation and release of these pollen, causing various allergic reactions, such as eczema and asthma, skin diseases and also add some new pathogenicity factors in the environment.

\section{The Mechanism of the Disease by These Particles}

In 2004 for the first time, The American Academy of Cardiology, stated that exposure to suspended particles contaminates the air cause the inability and mortality of cardiovascular disease. According to the available evidence, exposure to dust with a diameter of less than 2.5 micrometres in a few hours to several weeks increases the mortality and worsening of cardiovascular disease and prolonged contact (for example, one or two years), Same concept repeated the life expectancy of the population in contact with it for several years [3].

Airborne dust contaminants trigger inflammatory reactions, breakdown of the body's nervous system and deterioration of the arteries (deposition of fats in the body's veins) [13]. These particles also cause blood clots in the body's veins and strokes [10].

The American Lung association describes the effects of suspended particles: short-term effects (from several hours to days) including deaths from heart and lung diseases such as stroke, increased heart attacks, especially in middle-aged and those with heart disease. Inflammation of the lung tissue in young and healthy adults, increased hospitalization due to heart disease, increased appetite for emergency patients due to acute respiratory problems, increased incidence and severity of asthma in children, and long-term effects (annual) are increased hospitalization due to asthmatic attacks for children at a distance of 200 meters from highway. Another effect is the Reduced lung growth in children and adolescents which is a noticeable damage caused by to small and medium airways (airways less than $2 \mathrm{~mm}$ in diameter). This effect is similar to the effect of smoking in adults who have high cigarette smoking.

in addition, the set of all factors that determine the chemical composition and physical properties of the particles should be considered, such as the oxidation potential, solubility, electrical charge, particle size and shape, the number of particles, settling down or not in the lungs, and combining or not with other materials in the atmosphere or body tissues for these particles.

In fact, these are factors that determine the amount of particle toxicity, for example, the presence of transition metals (transitional elements or intermediates), organic compounds, liposaccharides (very toxic and pathogenic substances for the body), and microbes cause heart disease.

Many other pollutants, called secondary pollutants, mainly caused by the chemical reaction in the atmosphere between different pollutants. In detail, Sunlight, water vapour and clouds combine existing pollutants and create new and more destructive pollutants.

Today, more attention is paid for controlling and identifying that every pollutants are from each source and determine how harmful they are. Because it's ultimately the combination of all pollutants from an infectious source that affects human health and determines the outcome. For example, dust, mist and air pollutant microbes will have a much more destructive impact if combined with other pollutants, such as industrial smoke in industrial areas around cities. Studies also show that the decrease in suspended air particles has increased life expectancy in humans, for example by a decrease of 15 micrograms in particles with a diameter of 2.5 micrometers per cubic meter for a long time, the life expectancy mean increased by $0 / 02$ to $0 / 61$ years [8].

Other studies have shown that contact with particles with a diameter of less than $2.5 \mu \mathrm{m}$ disrupts the normal DNA replication and affects the function of the genes [1]. Also, these particles cause the loss of respiratory insufficiency in humans, which is the cause of various pulmonary diseases [4]. Other studies show that dust has the most danger and negative impact on poultry and honey bees. This phenomenon reduces the life of the bee, aphid decay and the occurrence of some complications due to disturbances in the direction of the bees due to the reduction of olfactory strength, and the loss of quality of pollen of flowers and nectar of plants, and consequently, loss of product quality and reduced production of its are other destructive effects [5]. 


\section{Other Destructive Effects of Suspended Particles and Germs}

In addition to pathogenicity, dust storms have other effects, including the burial of villages and places below the sand masses, the displacement of people in cities and their suburbs, and economic and social problems, increasing population density of cities, the loss of job opportunities, the increase in poverty and illegal activities such as fuel smuggling. Moving from a dry bed of lakes, followed by more destruction and erosion, filling of canals and raising the cost of dredging, rising riverbeds and blocking water pipes due to the arrival of sand and spoiling millions water cubic meter per year, huge economic damage to agricultural and production facilities, blockages of important communication roads, late arrivals of products to the market and reduced their quality, increased road accidents due to reduced visibility and many financial losses are some other problems of these particles.

As previously discussed, this study further emphasizes the impact of air pollution on human health, which increases the pollution poses a serious threat to the health of citizens who are in contact with these particles. Also, in dust storms, these particles cause corneal incisions and blindness. For example, statistics show that about 28 percent of the blindness of Sistan $\&$ Baluchestan province in Iran is due to dust storms and particulates [6].

So far, many studies have focused on the concentration of pollutants and their effects on human health worldwide. Studies show that the impact of pollutants on human health caused by the consumption of a product that exported to another country, even showed in the country of manufacture. Results show that the efforts of the producing countries alone to mitigate the human health effects associated with PM2.5 are insufficient, because consumer demand may continue to increase ([11], [12]). Developed countries make significant contributions not only to economic production but also to human health impacts on PM2.5 in Asia, especially in China and India. Therefore, in order to reduce the human health effects in relation to PM2.5 globally, cooperation efforts between producer and consumer countries is essential.

In another study in Beijing, release of PM2.5 is equivalent to losing health and examines the effects of these particles on health with different models. The results of this study show that with the controls on contamination in Beijing in 2014 and 2015, the level of disease caused by these particles has decreased significantly. Also, the effect of these pollutants is most in the winter while it is the less due to the effects of the climate in the summer [7].

In another study in China, dusts specially PM2.5 (PM2.5) has been introduced as an important Disruptive factor for health and fitness, and they believe that anti-air pollution policies should be adopted by all levels of government in China [14].

World Health Organization (WHO) reports on the global particle pollutant situation in May 2016 showed that, over $80 \%$ of the world's population are exposed to excessive air pollution. These statistics refer to those cities that condition of the air pollution has been measured in them. The database shows that cities with high population and low income levels are more exposed to air pollution than other cities in the world. WHO, in its latest survey, compared PM10 and PM2.5 Concentrations for 795 cities in 67 countries over the 2008-2013 period. The statistics showed that the global level of suspended particulate matter has increased by $8 \%$, while in some regions it has relatively improved. The highest levels of urban air pollution have occurred in low-income and middle-income countries in the eastern Mediterranean and south eastern Asia, with the annual average level of particulate matter in these areas often more than 5-10 times the WHO limit.

\section{Ways to Prevent Dust and Small Particles in the Air}

Usually, due to the lack of sufficient water in arid areas and high wind speeds, biological operations and planting of seedlings are not possible. For this reason, it is recommended to use the indigenous knowledge and experiences of people living in arid areas, as well as the opinion of the professors and researchers. Sometimes windbreaks in areas exposed to the wind are recommended to prevent the movement of sand. For set up a strong wind break it is better to use dams and shrubs that are resistant to drought in areas such as Haloxylon or Tamarix or their branches. The evacuation of gravel from the villages and emptying them in distant areas, mulching operations on hills, and to prevent the soil from sticking down and destroying for the hurricanes and sunshine, seedlings on the hills can be practical [6].

\section{Discussion and Conclusion}

As stated above, wind erosion, as one of the effective factors in desertification, gradually weakens fertile lands and, destroys their production capacities by decreasing the production capacity of biofuels. Wind erosion and dust have a huge impact on human health and cause various diseases. Considering that controlling these diseases with regard to their factors 
of preventing the contamination of the particles or reducing their levels is much easier and more practical than controlling and preventing these diseases from uncontrollable factors such as genetics and gender or etc. According to the results of the climate survey of the Khuzestan region can be concluded that occurrence of dust storm and erosion is before the peak temperature and rainfall; therefore, examined climatic factors are caused wind erosion Statistics highest effect in Abadan city and its impact is lowest in Dezful city. So nowadays the Issue of preventing these particles in the air and reducing the contact of people with these harmful substances in medicine is very significant. Based on what has been explained, we have to address to climate change, desertification, and especially wind erosion, as very effective factors in human health, caused by unethical and incorrect work for example there should be some strict rules for control exploitation of mines. Countries also should play a more effective role in managing and resolving this problem by maximizing interactions and making more precise decisions at regional and international meetings and working together as in many of these cases, the source of most particles and dust is in the neighboring countries. In addition, in the face of these storms, reinforced warning systems and air pollution control stations should be increased to prevent and dealing with these materials better than before.

\section{References}

[1] A. Baccarelli, R. Wright, V. Bollati, L. Tarantini, A. Litonjua, H. Suth, A. Zanobetti, D. Sparrow, D. S. Vokonas, I. Schwrtz, "Rapid DNA methylation changes after exposre to traffic particles Am J Respircirt care," Med, vol. 179, pp. 572-578, 2009.

[2] A. Salamt, M. Aleyasin, "Strategies to mitigate the effects of drought, Iranian Irrigation and Drainage Committee," 2011.

[3] Canadian Council of Minister of the Environment Fine Fraction of PM, Human Health Effects of Fine Particulate Matter. Update in Support of the Canada-Wide Standards for Particulate Matter and Ozone. 2005.

[4] D. Urich, S. Soberanes, "Proapoptptic Noxa is required for particulate matter-indused cell death and lung inflammation," Faseb J, 2009.

[5] K. Khaledi, "Economic losses of dust storms in western provinces of Iran (Case study: Ilam, Khuzestan and Kermanshah, Iran)," Economic Modeling Quarterly, no. 3-23, pp. 125-105, 2013.

[6] Gh. R. Noori, "Iran's desert and desert site, Hamoon, and the reason for alternate droughts in Iran," 2015.

[7] L. Li, Y. Lei, S. Wu, J. Chen, D. Yan, "The health economic loss of fine particulate matter (PM2.5) in Beijing," Journal of Cleaner Production, vol. 161, p. 1153e1161, 2017.

[8] M. Ezzati, D. W. Dockery, "Fine Particulate air pollution and life expectancy in the United States," N Engl J Med, Pope CA $3^{\text {rd }}$, vol. 360, pp. 376-378, 2009.

[9] N. Franklin, Jr. Atkinson, “al Middleton's Allergy Principles \& Practice - seventh edition,” Elsevier, 2009.

[10] R. Brook, S. Rajagopalan, "Particulate Matter Air Pollution and Cardiovascular Disease: An Update to the scientific statement from the American Heart Association," Journal of the American Heart Association, 2010.

[11] S. Liang, S. Guo, J. P. Newell, S. Qu, Y. Feng, A. S. F. Chiu, M. Xu, "Global drivers of Russian timber harvest," J. Ind. Ecol., vol. 20, p. 515e525, 2016.

[12] S. Liang, Y. Wang, S. Cinnirella, N. Pirrone, "Atmospheric mercury footprints of nations," Environ. Sci. Technol., vol. 49, p. 3566e 3574, 2015.

[13] US Environmental Protection Agency, National Ambient Air Quality Criteria Standards. 2008.

[14] J. Yu, C. Gao, J. Kai Wei Lee, J. Zhao, PM2.5: “A barrier to fitness and health promotion in China," Journal of Sport and Health Science, vol. 6, pp. 292-294, 2017. 